\title{
Fast Vibroacoustic optimization of mechanical structures using artificial neural networks
}

\author{
Mostafa Ranjbar ${ }^{1, *}$, Steffen Marburg ${ }^{2}$ \\ ${ }^{1}$ Department of Mechanical Engineering, Faculty of Engineering, Eastern Mediterranean University, Gazimagusa, Turkey \\ ${ }^{2}$ LRT4, Institut für Mechanik, Universität der Bundeswehr München, Neubiberg, Germany
}

Email address:

Mostafa.Ranjbar@emu.edu.tr(M. Ranjbar), Steffen.Marburg@unibw.de(S. Marburg)

To cite this article:

Mostafa Ranjbar, Steffen Marburg. Fast Vibroacoustic Optimization of Mechanical Structures Using Artificial Neural Networks. International Journal of Mechanical Engineering and Applications. Vol. 1, No. 3, 2013, pp. 64-68. doi: 10.11648/j.ijmea.20130103.11

\begin{abstract}
An artificial neural network (ANN) is adjusted to make analytical approximation of objective function for a specific structural acoustic application. It is used as the replacement of the main real objective function during the optimization process. The goal of optimization is to find the best geometry modification of the considered model which is supposed to produce lower values of the radiated sound power levels. The result of this study shows that the function approximation by neural networks can reduce the duration of optimization procedure. Furthermore, the tuning of ANN internal parameter settings is a real challenge to be considered.
\end{abstract}

Keywords: Design, Optimization, Vibroacoustic, Artificial Neural Networks, Mechanical Structures, Rectangular Plate

\section{Introduction}

Passive noise control describes methods to optimize structures numerically with respect to various acoustical and structural properties such as the root mean square level of the structural particle velocity, the radiated sound power etc. A survey of methods and applications of structural acoustic optimization for passive noise control can be found in the review paper by Marburg [1].

Neural network imitates a neurobiological process that processes input and generates output; it can be trained by pairs of input and output data. During this training period, weight factors are adjusted in the connections between certain nodes of the network so that the generated output matches expected output data. Once trained, the network is used as a simple function that substitutes for an original one. A technique for general subsequent usage of artificial neural networks (ANN) was proposed by Arslan et al. [2]. ANN method (NN) is more suitable for the applications in which is no way to describe the problem with an analytical function. A trained network presents a rapid mapping of given input into the desired output quantities, thereby enhancing the efficiency of the redesign process.

The ANN training comprises the following tasks. At first select the proper training set, then find the suitable network architecture and determine the appropriate values of characteristic parameters such as the learning rate and momentum term [3]. However, few applications of neural networks as approximation scheme are known for structural acoustics. Nagaya and Li [4] applied a three-layered neural network system for optimization of fifteen variables. Another example of neural networks utilized holographic neural network [5].

Kirkpatrick et al. [6] were the first to present the simulated annealing (SA) concept. This method is able to escape from local minima and find the global minimum [7, 8]. SA algorithm was adapted to problems with continuous variables by Corana et al. [9]. The adapted version was tested against an adaptive random search method and the Nelder and Mead simplex algorithm [10], using some of Rosenbrock's test functions [11]. Furthermore, a simulated annealing algorithm was used by Constans et al. [12] to minimize the sound power radiated from a vibrating shell structure. Also, Shim and Manoochehri [13] presented a computer-based shape configuration design methodology employing of SA method to generate optimum design of specified structures satisfying the structural performance requirements and the geometric connectivity of the model.

The problem which will be considered in the current paper is too complex to be solved analytically. Therefore, only a numerical solution is possible. An ANN is used to calculate a numerical model from the objective function. ANN trains a set of neurons to work as a replacement for the main objective function. For this purpose, a set of 
training members including the main objective function's values in several discrete design points are be considered. Then the neural network is being trained to simulate the main objective function virtually. Then the trained objective function can be calculated in a shorter time than other methods as well as finite elements analysis.

After careful review of previous works in the field of structural acoustic optimization, it is experienced that there is a lack of study on the combination of artificial neural networks as a virtual function approximation tool and simulated annealing method as an optimization tool in structural acoustics. The aim of this paper is to explore this open research area in structural acoustics. At this regard, the global approximation scheme ANN is used in combination with the simulated annealing method [14]. Furthermore, the results which are presented in this work stem from Ranjbar [15]. His work contains many more details and even comparison between additional methods which are not discussed in detail in this paper.

\section{Optimization Procedure}

A reliable measure for the noise emitted from the structure or machine part is the level of radiated sound power or sound power level, which is a function of circular frequency [16, $17,18]$. In this paper, only structure borne sound is considered. It means that there is no coupling between the structure and the surrounding fluid, i.e. Air. So, the air has no effects and induces no pressure on the surface of the model. Furthermore, the half-plan radiation is considered. Also, the radiation efficiency is considered as one.

The level of structure borne sound can be interpreted as a measure of the vibrational sensitivity of a structure when subjected to some excitation.

Since acoustic power is determined by the surface velocity, one alternative and less computationally expensive objective function is to consider only vibrational efficiency of the structure as expressed by the mean square normal velocity [19]. This is also known as the equivalent radiated sound power [20]. So, an equivalent radiated sound power level is considered as the objective function to be minimized.

The level of structure born sound (LS) constitutes a spectrum, i.e. it is a function of circular frequency. To obtain some single global measure of the vibrational behavior of a structure in a given frequency range of interest, the root mean square level of structure borne sound over that frequency band, known hereafter as RMSL [21], is calculated as

$$
R M S L=\sqrt{\frac{\int_{\omega_{\min }}^{\omega_{\max }} L S^{2}(\omega) d \omega}{\omega_{\max }-\omega_{\min }}}
$$

In Eq. (1), $\omega_{\max }$ and $\omega_{\min }$ are the lower and upper bounds of the circular frequency range under consideration, respectively. The RMSL is the root square of the area beneath the LS spectrum divided by the width of the frequency band and can be computed numerically. In this paper, the RMSL is considered as the objective function to be minimized.

The level of structure born sound (LS) constitutes a spectrum, i.e. it is a function of circular frequency $\omega$.

As it is indicated, a global measure of the vibration behavior of a structure is the root mean square level of structure borne sound over that frequency band, known RMSL.

The RMSL is the root square of the area beneath the LS spectrum divided by the width of the frequency band. Herein, the RMSL is the objective function (F) for the minimization.

The structure to be optimized is a square plate made of steel $[22,23]$. The damping is assumed to be independent of frequency with a constant damping coefficient of $0.3 \%$. The concept of geometry based modeling technique is followed [24].

Herein, the optimization problem is defined as follows

$$
F(v)=\operatorname{RMSL}(v) \rightarrow \min ,
$$

While the set of design variables remain in a prescribed interval of lower and upper modification values as

$$
-10 \mathrm{~mm} \leq v_{i} \leq 10 \mathrm{~mm}
$$

Equation (3) defines the design space just as a nine-dimensional cube since all parameters are allowed to take values within the same fixed interval and all parameters are independent of each other's. There are no additional equality and inequality constraints.

As mentioned above, the design variables, $Z_{i}(i=1,2, \ldots, 9)$, are the positions of specific points, in other words, the normal geometry modifications at these movable points. If the shape of the surface is varied by means of a spline function, then the positions of the spline points are the design variables.

Fig. 1 depicts the FE model. The geometry is defined by a square of $1 \mathrm{~m}$ edge length. Also, nine design key-points, i.e., $Z_{i}(i=1,2, \ldots, 9)$ are considered on the surface of the plate The plate is composed of 16 areas. Each area is meshed by $5 \times 5$ quadrilateral, eight node Serendipity shell elements [24], i.e. the plate's mesh consists of 400 finite elements. The plate is simply supported. It is $1 \mathrm{~mm}$ thick.

There are three uniform harmonic pressure excitations on the surface of plate which are shown with the hatched areas in Fig. 1. All of them act at the same amplitude and phase and are uniform over the frequency range of $0-100 \mathrm{~Hz}$. The excitations pressures act at the locations where presumably all relevant mode shapes of the structure in the frequency range of interest are excited. 


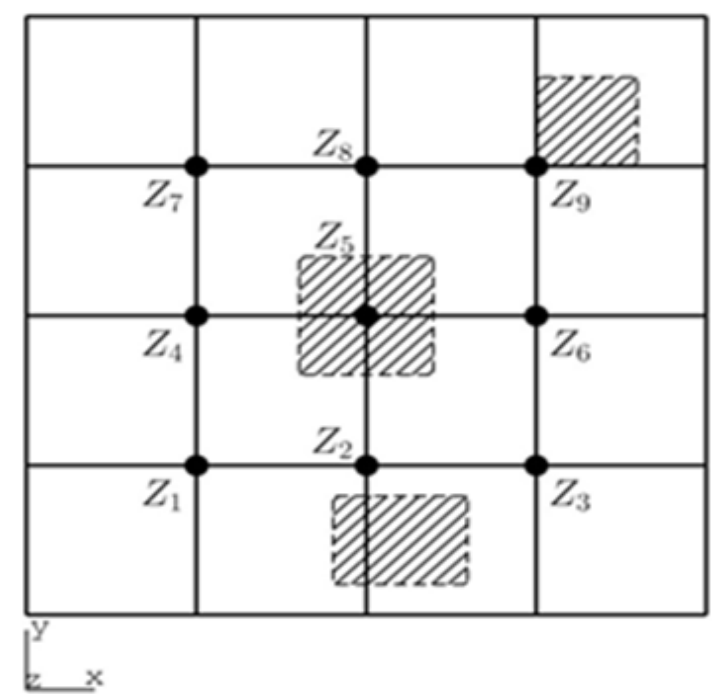

Figure 1. Initial model of the rectangular plate with three harmonic excitations (hatched areas) and nine design points connected with splines to each other's.

The optimization method basically works as follows: build initial approximation by ANN based on a given sample set which were calculated by previous experiments, use optimization method to find the minimum of the approximated objective function to get a new design, evaluate the new design with the full analysis code, use the new results to improve the accuracy of the optimum designs and continue until termination criterion is met. The main termination criterion used in this study is the maximum number of function evaluations, e.g. 500 function evaluations.

In this paper an ANN is used to be trained for the calculation of objective function. The objective function values produced by $\mathrm{ANN}$ are used for the optimization process by SA method. Indeed, it is a combination of ANN method as the function approximation tool and method of Simulated Annealing as the optimizer tool.

The considered neural network has five hidden neurons and uses the back propagation learning algorithm. Initial and final learning rates are 0.1 and 0.01 . ANN using an initial training set of 110 function samples. Training loop is repeated for 100 times. The optimization iterations are continued up to 500 times. However, this number of function evaluations is no problem as it takes actually a very short time. A back propagation learning algorithm with five hidden neurons is used. Initial and final learning rates are 0.1 and 0.01 in order. The ANN uses an initial training set of 110 function values. Then, an initial 110 minutes computation time must be invested for the calculation of training set.

There are a lot of possibilities to consider different combinations of different larger or smaller training sets, different number of training loops, the number of neurons and layers and input-output learning rates.

Choosing the best parameters for a good neural network needs itself a separate study and even with try and error approach.

SA method is employed as the optimization tool. It uses the calculated approximate function values by ANN method for the minimization process. The required parameters for SA solver remain unchanged as explained in ref. [8] and are similar with the case when the SA solver works as usual to find the minimum of an objective function. Just the user must provide an initial start design for SA solver and the maximum allowable number of objective function evaluations.

Tuning of suitable initial parameters for optimization process needs a try and error approach and is beyond of scope of this study. Therefore, the basic and previously recommended initial settings for ANN in ref. [25] are used to avoid the excessive effort for finding a well-tuned set of initial parameters.

The minimum required number of function evaluations for HNN method is variable. It is depended on the type of objective function approximation by this method.

\section{Optimization Results}

The root mean square of the radiated sound power level RMSL of the initial rectangular plate in the frequency range of interest $0-100 \mathrm{~Hz}$ is $42.7 \mathrm{~dB}$, where the maximum radiated sound level of $80.56 \mathrm{~dB}$ can be found in the spectrum at the fundamental frequency $4.9 \mathrm{~Hz}$. The structural mass of the initial structure can easily be determined analytically.

All of the quantities listed in the table 1 can basically serve as either objective function or constraint for the optimization calculations. In this paper, as it is mentioned before, the root mean square of the radiated sound power level (RMSL) is considered as the objective function for the optimization process.

The LS spectrum of the original and initial designs is shown in Fig. 2 The maximum LS of the optimized structure are decreased to $58.9 \mathrm{~dB}$ at the new fundamental frequency. The LS value is almost decreased in most of the frequency domain. This considerable reduction in the value of maximum LS is mainly caused by a suitable control parameter setting in algorithm. The proposed optimization approach has reduced the RMLS by about $9.6 \mathrm{~dB}$, has maximized the first natural frequency by $9.9 \mathrm{~Hz}$, has decreased the maximum LS by about $10.2 \mathrm{~dB}$, and has taken around 1.86 hours (CPU time) to perform 110 function evaluations for an optimization attempt. The optimization results for $\mathrm{HNN}$ are summarized in Table 1.

Table 1. Summary of optimization results

\begin{tabular}{lll}
\hline & Initial Design & Final Design \\
\hline RMSL & $42.7 \mathrm{~dB}$ & $33.1 \mathrm{~dB}(-9.6 \mathrm{~dB})$ \\
Minimum modification & $-7.58 \mathrm{~mm}$ & $-3.258 \mathrm{~mm}$ \\
Maximum modification & $8.625 \mathrm{~mm}$ & $9.850 \mathrm{~mm}$ \\
Fundamental frequency & $21.8 \mathrm{~Hz}$ & $32.8 \mathrm{~Hz}(+11.1 \mathrm{~Hz})$ \\
Maximum LS & $69.1 \mathrm{~dB}$ & $54.9 \mathrm{~dB}(-14.2 \mathrm{~dB})$ \\
\hline
\end{tabular}




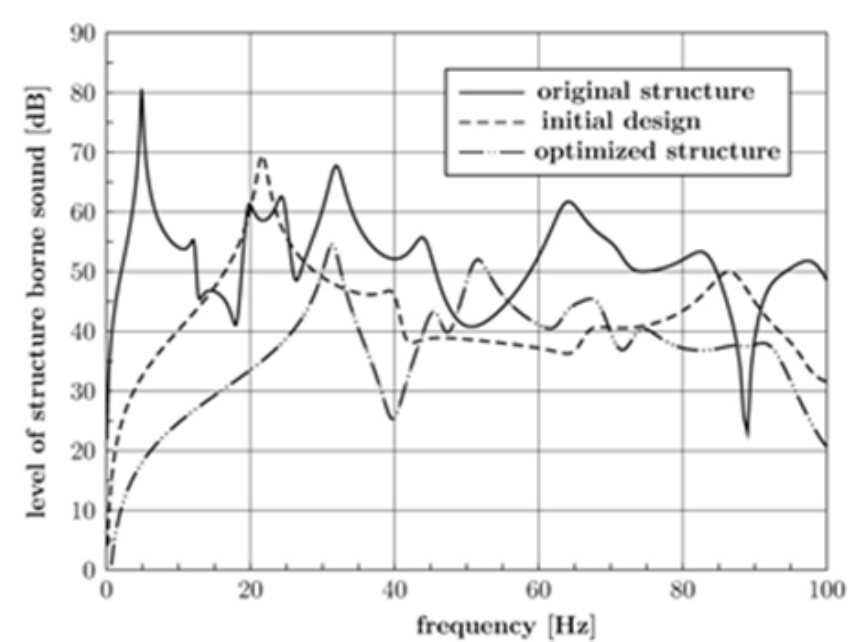

Figure 2. LS spectra of the original and the optimized rectangular plate by $H N N$

The LS spectrum in Fig 2 shows that there are resonance frequencies in the range of $0-100 \mathrm{~Hz}$ for the original flat plate. It is reduced around five in the initial design which has some geometry modifications. Furthermore, it is shown that the number of resonance frequencies for the final optimum design is relatively similar with initial design case. However, it seems that a finer frequency discretization should be used in the range of 40-60 Hz, too.

The contour plot of the plate's optimized geometry is shown in Fig. 3. The optimized geometry distribution can also be interpreted as a stiffening rib across the diagonal of the plate, which efficiently suppresses vibrations.

The minimum and maximum geometry modifications, see Fig.3, are -3.259 and 9.850 millimeters, respectively. It is visible that the modified geometry, will affect most of vibrating mode shapes. In fact, they are located in the areas which under pressure excitations.
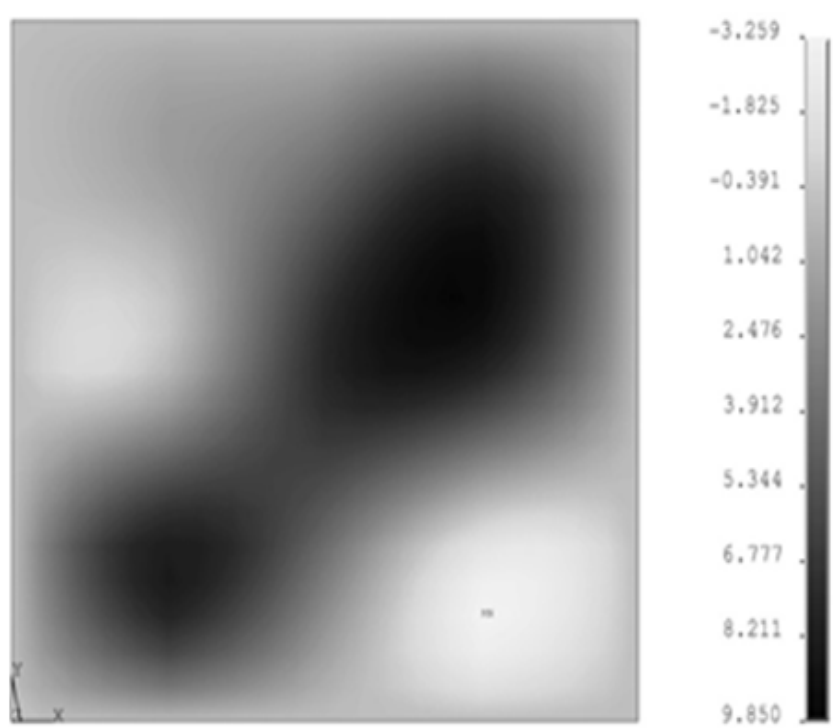

Figure 3. Geometry distribution of the modified rectangular plate (values in $\mathrm{mm}$ ).

\section{Conclusions}

It has been shown that the optimization procedure used for this study is able to produce significant improvements of the objective function. This method can be used for numerical optimization in structural acoustics and it can be expected to achieve acceptable results after a certain number of objective function evaluations.

The presented method is able to reduce the RMSL of objective function.

Since the tuning of initial parameters for ANN has been done in a tray and error manner, it is actually not possible to make a general conclusion about the convergence rate.

There are still several issues remained to be investigated. Other model shapes rather than rectangular should be examined. Furthermore, different types of model materials, e.g. composites and other metals may be tested.

The calculation of virtual objective function values by the trained $\mathrm{NN}$ is very fast. It takes just a few milliseconds. Therefore, in comparison with other methods, ANN is relatively fast and can produce acceptable optimization results. However, designing of an efficient NN depends on the type of the problem and the experience of the user and sometimes with a trial and error approach.

Performing of a sensitivity analysis of ANN for the various initial parameter settings will be necessary for the future works. Indeed, it is necessary to do more researches in this field.

\section{References}

[1] St. Marburg, "Developments in Structural-Acoustic Optimization for Passive Noise Control," Archives of Computational Methods in Engineering. State of the art reviews, vol. 9, 2002, pp. 291-370.

[2] M. A. Arslan, and P. Hajela, "Use of Counterpropagation Neural Nnetworks to Enhance the Concurrent Subspace Optimization Strategy," Engineering Optimization, vol. 33, 2001, pp. 327-349.

[3] K. H. Baek, and S. J. Elliott, "Natural Algorithms for Choosing Source Locations in Active Control Systems," Journal of Sound and Vibration, vol. 186 (2), 1995, pp. 245-267.

[4] K. Nagaya, and L. Li, "Control of Sound Noise Radiated from a Plate using Dynamic Absorbers under the Optimization by Neural Network,"Journal of Sound and Vibration, vol. 208, 1997, pp. 289-298.

[5] Q. Shi, I. Hagiwara, A. Azetsu, and T. Ichkawa, "Holographic Neural Network Approximations for Acoustic Optimization,” JSAE Review, vol. 19, 1998, pp. 361-363.

[6] S. Kirkpatrick, C. D. Jr. Gellat, and M. P. Vecchi, “ Optimization by Simulated Annealing," Science, vol. 220, 1983, pp. 671-680.

[7] W. Goffe, G. D. Ferrier, and J. Rogers, "Global Optimization of Statistical Functions with Simulated Annealing," Journal of Econometrics, vol. 60 (1-2), 1994, pp. 65-99. 
[8] W. Goffe, "SIMANN: A Global Optimization Algorithm Using Simulated Annealing," Studies in Non-linear Dynamics and Econometrics, vol. 1 (3), 1996, pp. 169-176.

[9] A. Corana, M. Marchesi, C. Martini, and S. Ridella, "Minimizing Multimodal Functions of Continuous Variables with the Simulated Annealing Algorithm," ACM (Association for Computing Machinery) Transactions on Mathematical Software, vol. 13 (3), 1987, pp. 262-280.

[10] J. A. Nelder, and R. Mead, " A Simplex Method for Function Minimization,"The Computer Journal, vol. 7 (4), 1965, pp. 308-313.

[11] H. H. Rosenbrock, “ An Automatic Method for Finding the Greatest or Least Value of a Function," The Computer Journal, vol. 3 (3), 1960, pp. 175-184.

[12] E. W. Constans, G. H. Koopmann, and A. D. Belegundu, "The Use of Modal Tailoring to Minimize the Radiated Sound Power of Vibrating Shells: Theory and Experiment," Journal of Sound and Vibration, vol. 217 (2), 1998, pp. $335-350$.

[13] P. Y. Shim, and S. Manoochehri, "A Hybrid Deterministic/Stochastic Optimization Approach for the Shape Configuration Design of Structures," Structural and Multidisciplinary Optimization, vol. 17 (2-3), 1999, pp. 113-129.

[14] M. Ranjbar, St. Marburg, and H.-J. Hardtke, "Development of a Hybrid Neural Networks Algorithm for Structural-Acoustics Optimization Applications", In Proceedings of the First International Conference of Acoustics and Vibration, 21-22 December 2011, Tehran, Iran.

[15] M. Ranjbar, A Comparative Study on Optimization in Structural Acoustics, $\mathrm{PhD}$ Thesis, Technische Universität Dresden, Germany, 2011.

[16] G. H. Koopmann, and J. b. Fahnline, Designing Quiet Structures: A Sound Power Minimization Approach, Academic Press, San Diego, London, 1997.
[17] F. G. Kollmann, Maschinenakustik--Grundlagen, Meßtechnik, Berechnung, Beeinflussung, in German, 2nd revised edition, Springer-Verlag, Berlin, Heidelberg, 2000.

[18] S. Marburg, "Efficient Optimization of a Noise Transfer Function by Modification of a Shell Structure Geometry. Part I: Theory," Structural and Multidisciplinary Optimization, Vol. 24, 2002. pp. 51-59.

[19] D. Fritze, St. Marburg, and H.-J. Hardtke, "Estimation of Radiated Sound Power: A Case Study on Common Approximation Methods," Acta Acustica united with Acustica, vol. 95, 2009, pp. 833-842.

[20] Ranjbar, H.-J. Hardtke, D. Fritze, and St. Marburg, "Finding the Best Design within Limited Time: A Comparative Case Study on Methods for Optimization in Structural Acoustics," Journal of Computational Acoustics, vol. 18 (2), 2010, pp. 149-164.

[21] M. Ranjbar, St. Marburg, and H.-J. Hardtke, "Structural-Acoustic Optimization of a Rectangular Plate: A Tabu Search Approach," Journal of Finite Elements in Analysis and Design, vol. 50, 2012, pp. 142-146.

[22] M. Ranjbar, and St. Marburg, “ Vibroacoustic Optimization of Mechanical Structures: A Controlled Random Search Approach," Advanced Materials Research, vol. 623, 2013, pp. 158-161.

[23] St. Marburg, "Efficient Optimization of a Noise Transfer Function by Modification of a Shell Structure Geometry. Part I: Theory," Structural and Multidisciplinary Optimization, vol. 24, 2002, pp. 51-59.

[24] ANSYS ${ }^{\circledR}$ Academic Research, Release 11.0, Help System, ANSYS, Inc.

[25] M. Papadrakakis, N. D. Lagaros, and Y. Tsompanakis, "Optimization of Large-Scale 3-D Trusses Using Evolution Strategies and Neural Networks," Special Issue of International Journal of Space Structures, vol. 14, 1999, pp. 211-223. 